\title{
Significance of nuclear morphometry as a diagnostic tool in fine-needle aspirates of breast masses
}

\author{
Indrani Krishnappa ${ }^{1}$, Raja Parthiban ${ }^{2, *}$, Akanksha Sharma ${ }^{3}$, Pooja Rani ${ }^{4}$ \\ ${ }^{1}$ Assistant Professor, ${ }^{2}$ Professor, ${ }^{3,4}$ Post Graduate, Dept. of Pathology, MVJ Medical College \& Research Hospital, Bangalore, \\ Karnataka, India
}

*Corresponding Author: Raja Parthiban

Email: indianpathology@gmail.com

Received: $29^{\text {th }}$ April, 2018

Accepted: $26^{\text {th }}$ June, 2018

\begin{abstract}
Breast carcinoma ranks one among the leading causes of cancer related morbidity and mortality in Indian women closely following carcinoma cervix in order of incidence. Fine needle aspiration cytology has emerged as one of the preliminary investigations besides mammography and clinical examination for the initial assessment of breast masses. Nuclear morphometry can be used as a quantitative adjuvant to FNAC in diagnosing breast lesions, especially the ones in the "gray zone".

Objectives: The aim of this study was to assess the utility of nuclear morphometry as an adjuvant in the cytological diagnosis, categorization of breast lesions and grading of malignant lesions.

Materials and Methods: Nuclear parameters were assessed in fine needle aspirates of 49 cases, for which histopathological correlation was available. Morphometric parameters analyzed were nuclear area, nuclear perimeter, minimal nuclear diameter, maximum nuclear diameter, axis ratio, nuclear shape factor and nuclear compactness.

Results: Morphometric parameters showed progressive and significant increase in nuclear size parameters from benign to malignant lesions. Nuclear shape parameters also showed a significant increase from grade I to grade III malignant lesions. However these shape factors could not be used to differentiate benign and malignant lesions in our study.

Conclusion: Nuclear morphometry is a valuable tool in diagnosis of breast masses. It provides data in a more objective and reproducible form unlike the conventional cytological analysis.
\end{abstract}

Keywords: Breast lesions, FNAC, Nuclear morphometry.

\section{Introduction}

The incidence of carcinoma breast has been increasing at alarming rates in the yesteryears in developing countries like India. Rapidly changing lifestyle and westernization of our dietary habits have been hypothesized to be the reasons for this raise in the breast cancer cases. FNAC has gained popularity as one of the preliminary investigations for evaluation of breast masses besides mammography and clinical examination as it is a non invasive procedure. However cytological observations are relatively subjective with substantial inter and intraobserver variations. ${ }^{1}$ This can lead to inaccurate results especially in cytologically overlapping lesions like atypical ductal hyperplasia and low grade ductal carcinoma breast. ${ }^{2}$

Morphological changes in the nuclear structure mark the beginning of cancer process. ${ }^{3}$ Nuclear morphometry is a useful means used for measurement of these structural changes in the nucleus by image analysis Nuclear morphometric analysis is an objective tool for measuring the nuclear size, shape, texture and density parameters. These parameters can be of diagnostic help in further grading of breast tumors as well. ${ }^{4}$

\section{Aims and Objectives}

1. To assess the utility of nuclear morphometric parameters in categorization of breast lesions on cytologic samples.

2. To assess the significance of these parameters in cytological grading of malignant breast aspirates.

\section{Materials and Methods}

The present study was conducted at a rural tertiary care hospital in South Karnataka for a period of one year, from Jan 2017 to Dec 2017. FNAC was performed on 132 patients who presented with breast lump during this period. Histopathological correlation was available for 49 cases which were included in this study. Corresponding FNAC smears stained with Hematoxylin and eosin with adequate material were used for analyzing the nuclear morphometric parameters. Morphometric analysis was performed on 50 nonoverlapping nuclei per case using Image J software (Fig. 1). Images were generated by Olympus camera linked to Olympus CX31 microscope at a total magnification of $100 \mathrm{x}$ at same setting of microscopic light intensity, iris diaphragm, and condenser position. The saved images were opened in Image $\mathbf{J}$ analysis software and nuclear parameters measured. "Modified Neubaur's chamber" was used for calibration before taking the measurements. Nuclear parameters evaluated are mentioned in Table 1. 


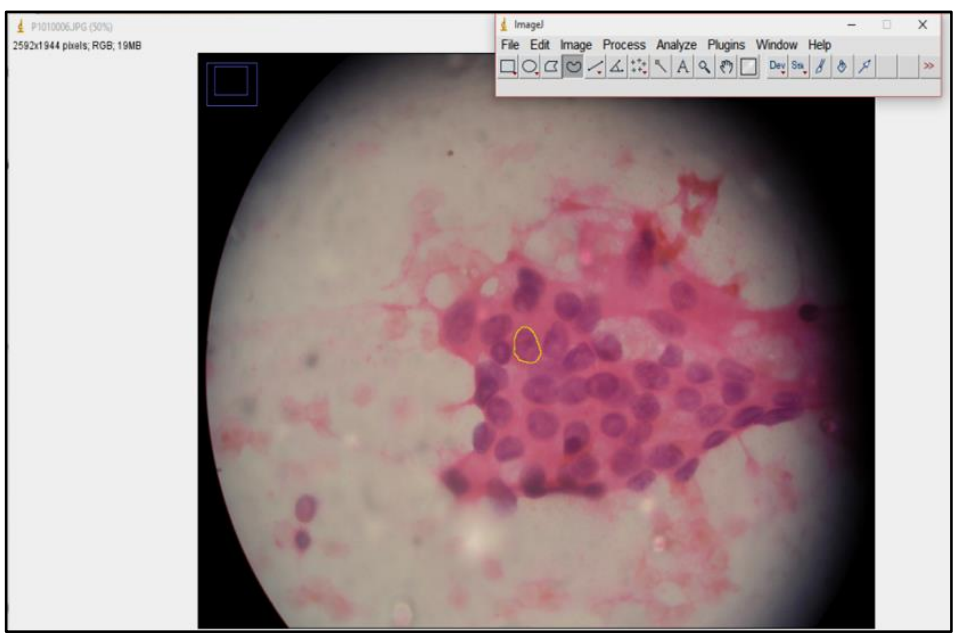

Fig. 1: Photo micrograph of an FNAC sample (100x magnification). Rounding of the nuclear boundary and measurement of nuclear parameters using Image J software.

Table 1: Nuclear morphometric parameters analyzed

\begin{tabular}{|l|c|}
\hline \multicolumn{1}{|c|}{ Parameters Measured by the Software } & Parameters calculated Manually \\
\hline Mean Nuclear Area (MNA) & Mean axis ratio (MAR) $=$ MmNd/MMND \\
\hline Mean Nuclear Perimeter (MNP) & Mean nuclear compactness (MNC)=MNP2/MNA \\
\hline Mean minimum Nuclear Diameter (MmND) & Mean nuclear shape factor \\
& $(\mathrm{MNSF})=4 \times \pi \times$ MNA/MNP2 \\
\hline Mean Maximum Nuclear Diameter (MMND) & \\
\hline
\end{tabular}

Aspirates from malignant lesions were graded according to "Robinson's cytological grading", grade-I (Score: 1-11); grade-II (Score: 12- 14); grade-III (Score: 15-18). ${ }^{6}$ Subsequently nuclear morphometric parameters were analyzed and compared between the three grades.

\section{Statistical Analysis}

The parameters obtained by computerised nuclear morphometry (Table 1) were compared between benign and malignant groups. The mean values of each sample with standard deviation (SD) were evaluated for the two groups. Statistical analysis was performed between the three malignant grades and $p$ value was calculated using Student's-t test and one-way ANOVA. p-value $<0.05$ were considered to be significant. The statistical correlations of the analyzed morphometric parameters with their variability for all samples; and cytological grades of all malignant samples were investigated.

\section{Results}

Based on cytological features $25(51.02 \%)$ cases were categorized as benign and $24(48.98 \%)$ as malignant. The 25 benign cases included 16 cases of fibroadenoma, 8 cases of fibrocystic disease and 1 case of benign papillomatosis. The 24 malignant cases included 23 cases of invasive ductal carcinoma and 1 case of lobular carcinoma. All these diagnoses were confirmed on histopathology.

Table 2: Comparison of nuclear morphological parameters between benign and malignant breast lesions

\begin{tabular}{|l|c|c|c|c|c|c|}
\hline \multirow{2}{*}{ S. No. } & Morphological parameters & \multicolumn{2}{|c|}{ Benign } & \multicolumn{2}{c|}{ Malignant } & p value \\
\cline { 3 - 6 } & Mean & SD & Mean & SD & \\
\hline 1 & Mean nuclear area (MNA) & 107.51 & 35.62 & 298.86 & 112.7 & $<0.0001$ \\
\hline 2 & Mean nuclear Perimeter (MNP) & 38.32 & 6.29 & 63.62 & 14.74 & $<0.0001$ \\
\hline 3 & Mean nuclear shape factor (MNSF) & 0.898 & 0.029 & 0.93 & 0.28 & 0.569 \\
\hline 4 & $\begin{array}{c}\text { Mean minimal nuclear diameter } \\
\text { (MmND) }\end{array}$ & 23.78 & 6.48 & 20.97 & 4.75 & 0.091 \\
\hline 5 & $\begin{array}{c}\text { Mean maximum nuclear diameter } \\
\text { (MMND) }\end{array}$ & 37.52 & 6.86 & 39.16 & 4.63 & 0.3337 \\
\hline 6 & Mean Axis ratio (MAR) & 0.625 & 0.09 & 0.543 & 0.071 & 0.0003 \\
\hline 7 & Mean nuclear compactness (MNC) & 13.99 & 0.47 & 14.12 & 2.09 & 0.763 \\
\hline
\end{tabular}


The means of nuclear area, nuclear perimeter and axis ratio were significantly higher in malignant cases. However nuclear shape factor and nuclear compactness values of benign lesions did show a statistical difference from those of malignant lesions. (Table $2 \&$ Fig. 2)
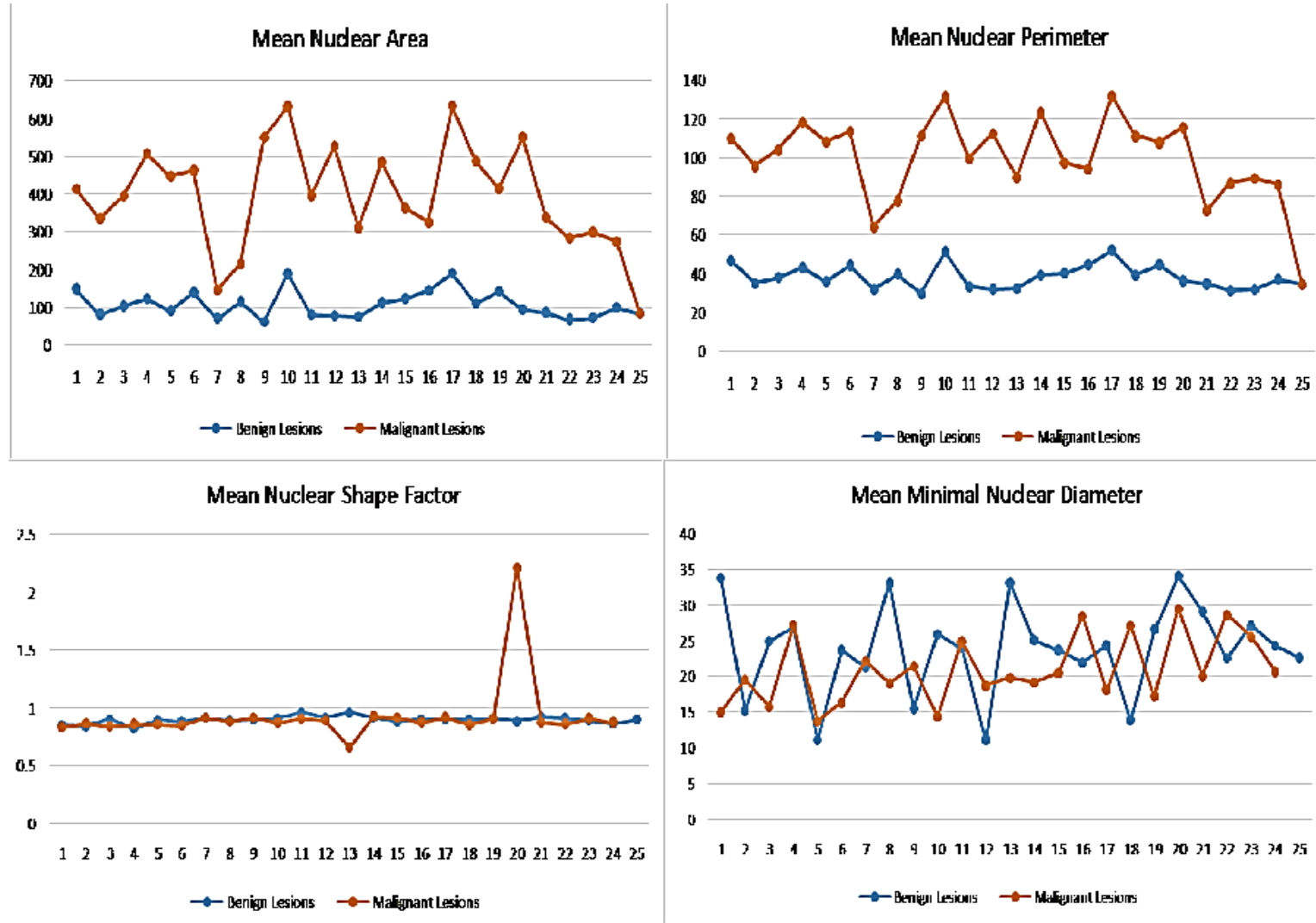

Mean Axis ratio
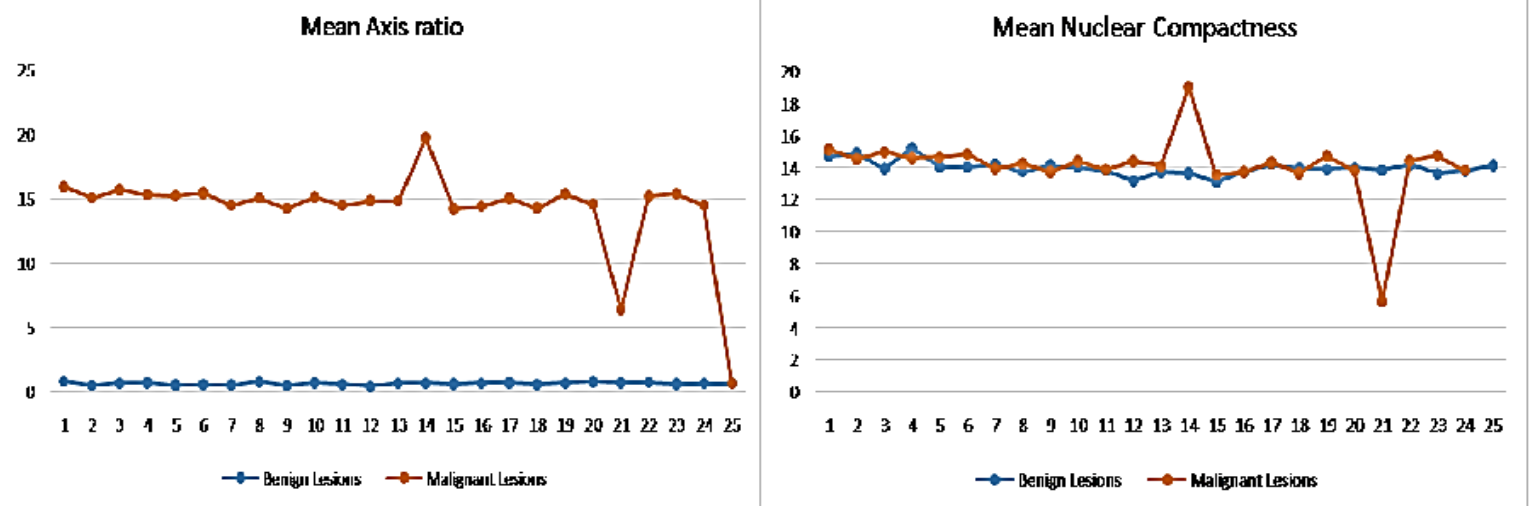

Fig. 2: Comparision of nuclear morphometric parameters between benign and malignant breast lesions. MNA, MNP \& MAR show significant difference between the two categories. The low MNA \& MNP for lobular carcinoma is reflected as a dip in the red line (case 7) in first two graphs

\section{Benign Lesions: (Fig. 3)}

The most commonly encountered benign lesion in our study was fibroadenoma (16 cases) followed by fibrocystic change (8 cases) and one case of benign

papillomatosis. The MNA in these cases ranged from 71.81 to $143.13 \mu$, MNP varied between 32.03 and 44.61 $\mu$ and the MAR ranged from 0.535 to $0.715 \mu$. 

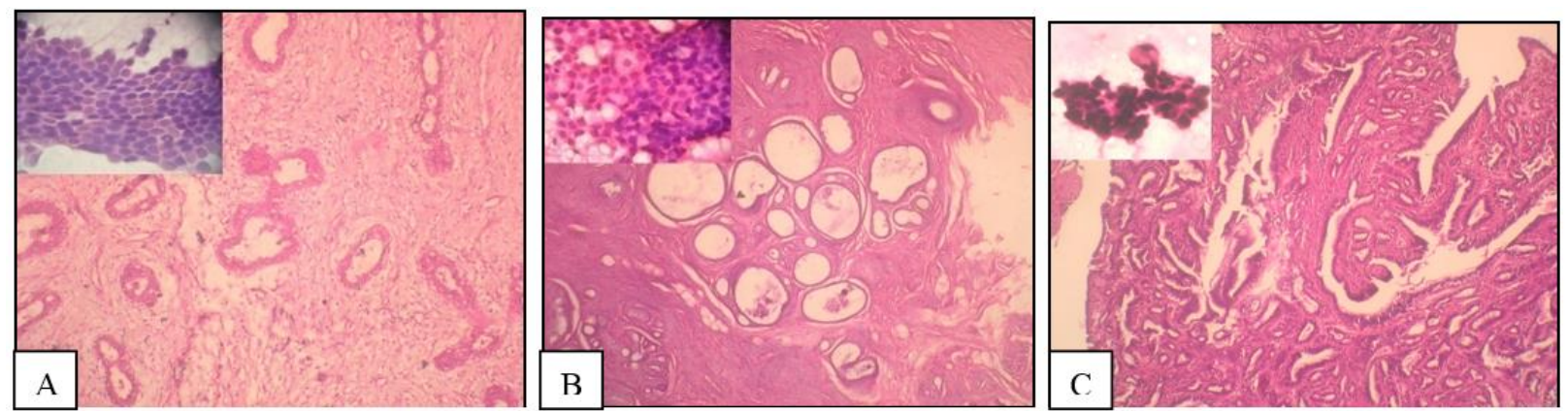

Fig. 3: A. Fibroadenoma (Inset - FNAC Fibradenoma); B. Fibrocystic change (Inset - Benign ductal epithelial cells); C. Benign papillomatosis (Inset - benign ductal epithelial cells in papillary configuration

\section{Malignant lesions: (Fig. 4, 5 \& 6 )}

Ductal carcinoma was the most common malignancy encountered in our study (23 cases) and we had one case of lobular carcinoma breast. The MNA in these lesions ranged between 186.16 and $411.56 \mu$, MNP varied between 48.88 and $78.36 \mu$ and the MAR ranged between 0.472 and $0.614 \mu$. The nuclear morphometric parameters were very low in case of lobular carcinoma breast compared to ductal carcinoma. MNA in lobular carcinoma breast (Table 3) was a mere $74.4 \mu$, MNP was $32.19 \mu$, MAR was $0.56 \mu$, MNC was 13.93 and MSF was 0.9 . These values were closer to the mean nuclear parameters of benign lesions.

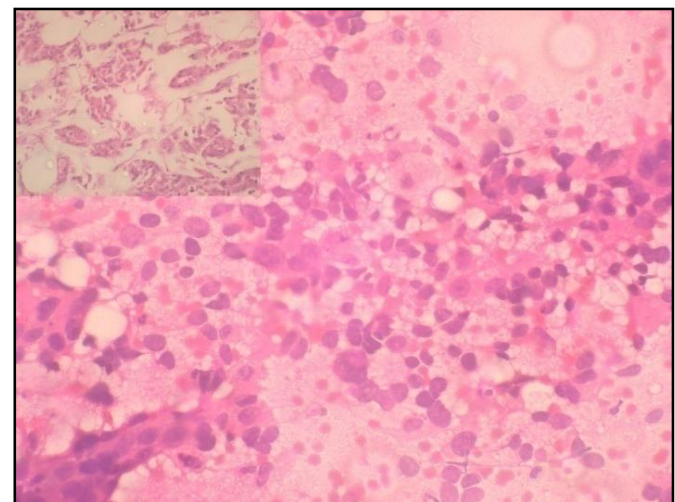

Fig. 4: Ductal carcinoma, grade I (Inset corresponding histopathology), 40X, H\&E

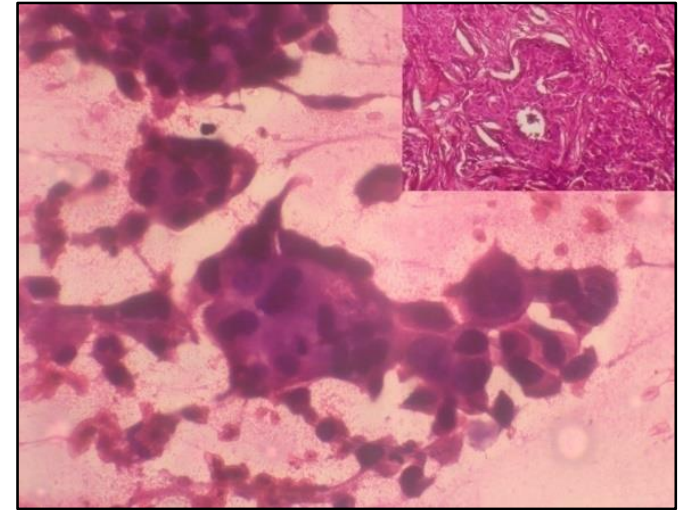

Fig. 5. Ductal carcinoma, grade II (Inset corresponding histopathology), 40X, $\mathrm{H} \& \mathrm{E}$

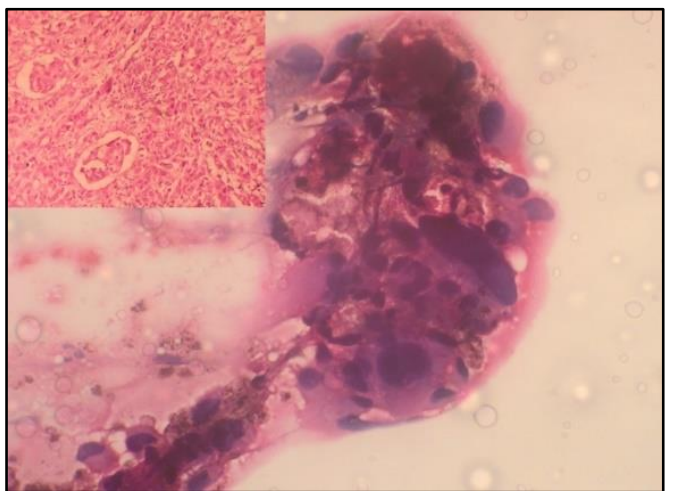

Fig. 6: Ductal carcinoma, grade III (Inset corresponding histopathology), $40 \mathrm{X}, \mathrm{H} \& \mathrm{E}$

Table 3: Comparison of Nuclear Morphological Parameters between benign breast lesions, IDC and Lobular Carcinoma

\begin{tabular}{|l|c|c|c|}
\hline \multicolumn{1}{|c|}{$\begin{array}{c}\text { Morphological } \\
\text { parameters }\end{array}$} & Benign lesions & IDC & $\begin{array}{c}\text { Lobular } \\
\text { Carcinoma }\end{array}$ \\
\hline Mean nuclear area (MNA) & 107.51 & 298.86 & 74.41 \\
\hline Mean nuclear Perimeter (MNP) & 38.32 & 63.62 & 32.19 \\
\hline Mean nuclear shape factor (MNSF) & 0.898 & 0.93 & 0.9 \\
\hline Mean minimal nuclear diameter(MmND) & 23.78 & 20.97 & 22.24 \\
\hline Mean maximum nuclear diameter(MMND) & 37.52 & 39.16 & 39.88 \\
\hline Mean Axis ratio (MAR) & 0.625 & 0.534 & 0.556 \\
\hline Mean nuclear compactness (MNC) & 13.99 & 14.12 & 13.93 \\
\hline
\end{tabular}


Robinson's cytological grading was applied on these cases and results of morphometric analysis of these cases was tabulated. (Table 4)

Table 4: Comparison of Nuclear Morphological Parameters between Robinson's Grade I vs Grade II and Grade II vs Grade III malignant breast lesions

\begin{tabular}{|c|c|c|c|c|c|c|c|c|c|c|}
\hline \multirow[t]{2}{*}{ Parameters } & \multicolumn{2}{|c|}{$\begin{array}{l}\text { Grade I (14 } \\
\text { cases) }\end{array}$} & \multicolumn{2}{|c|}{$\begin{array}{l}\text { Grade II (4 } \\
\text { cases) }\end{array}$} & \multirow[t]{2}{*}{$p$ value } & \multicolumn{2}{|c|}{$\begin{array}{c}\text { Grade II (4 } \\
\text { cases) }\end{array}$} & \multicolumn{2}{|c|}{$\begin{array}{c}\text { Grade III (6 } \\
\text { cases) }\end{array}$} & \multirow[t]{2}{*}{ p value } \\
\hline & Mean & SD & Mean & SD & & Mean & SD & Mean & SD & \\
\hline MNA & 266.86 & 31.11 & 318.97 & 69.93 & 0.0353 & 318.97 & 69.93 & 365.17 & 14.84 & 0.388 \\
\hline MNP & 59.64 & 15.86 & 66.11 & 14.52 & 0.033 & 66.11 & 14.52 & 323.42 & 1.34 & $<0.0001$ \\
\hline MNSF & 0.887 & 0.027 & 0.972 & 0.416 & 0.507 & 0.972 & 0.416 & 0.88 & 0.043 & 0.769 \\
\hline MmND & 23.03 & 4.125 & 18.997 & 4.111 & 0.033 & 18.997 & 4.111 & 20.43 & 9.41 & 0.707 \\
\hline MMND & 42.291 & 4.083 & 36.984 & 3.378 & $<0.0001$ & 36.984 & 3.378 & 39.43 & 10.53 & 0.496 \\
\hline MAR & 0.55 & 0.06 & 0.516 & 0.733 & 0.879 & 0.516 & 0.733 & 0.5 & 0.1 & 0.977 \\
\hline $\mathrm{MNC}$ & 14.177 & 0.432 & 14.066 & 3.131 & 0.908 & 14.066 & 3.131 & 14.16 & 0.104 & 0.968 \\
\hline
\end{tabular}

\section{Discussion}

FNAC is a non invasive and inexpensive investigation modality and therefore has been widely used to assess breast lumps initially on an outpatient basis. However, it can be associated with considerable degree of interobserver variability. Variations in cellularity, cell morphology, nuclear features and chromatin contribute to the subjective nature of this test. Also, it is not much useful in cases with morphological overlap that is the gray zones.

The current study evaluates the role of nuclear morphometry as an adjuvant to FNAC in accurate distinction between benign and malignant lesions preoperatively. Most of the studies on nuclear morphometry have been done on histopathological sections; however we are of the opinion that cytological specimens are better for morphometric analysis when compared to histopathology sections as the cells are better preserved in FNAC smears. Also histopathological processing induces numerous artifacts in the sections at various steps starting from fixation to processing and staining.

Benign v/s Malignant Lesions: Our study showed that benign and malignant lesions can be differentiated with a considerable degree of accuracy using nuclear morphometric analysis (Table 2). The size parameters i.e. MNA, MNP and MAR showed significant differences between benign and malignant breast lesions $(p=<0.05)$. The line graphs plotted for these parameters also showed wide gaps in the plot (Fig. 2). However, the shape factors revealed no significant difference between benign and malignant cases and showed overlapping of the plots (Fig. 2). These observations were consistent with the findings of other authors. ${ }^{7,8}$

Nuclear area is the most studied parameter by various authors. The current study also demonstrates significant difference in the MNA of benign and malignant lesions. Like Abdalla et al, our study also witnessed high SD for MNA of malignant lesions as compared to that of benign lesions attributing to higher degree of pleomorphism noted in malignant cases. ${ }^{9}$

Boon et al. preferred to use the nucleus/cytoplasm ratio for characterizing cells of different tumors. However, we feel such a design should be avoided because cellular outline is much indistinct than nuclear outline and it becomes very difficult to mark the cellular outline accurately, hence making the values and results more subjective and less reproducible. ${ }^{10}$

Unlike our study, a study done by Kashyap et al found even shape and texture factors to be significant in differentiating between benign and malignant lesions. ${ }^{11}$ However our results were in line with other studies of Kalhan et al, Aggarwal et al, Abdalla et al and Parmar D et al. ${ }^{7-10}$ Kashyap et al used Nikon Imaging Software-AR for their study, this is a relatively superior software which could measure the nuclear density and textures more accurately. These are significant parameters to differentiate benign from malignant lesions. This might be the reason for discordance from our study.

Nuclear morphometry acts as an auxiliary tool in accurately distinguishing cytologically overlapping lesions like benign proliferative lesions and low grade ductal carcinoma. Cytology in both these lesions shows minimal nuclear atypia and cellular dyscohesion. Low grade ductal carcinoma breast show cytological features similar to benign lesions like fibrocystic change and papillary lesions, these can be distinguished with some certainty with the use of nuclear morphometric analysis. ${ }^{13}$

Nuclear Morphometry Parameters \& Cytological Grading: Some authors have studied the correlation between morphometry and cytologic grading using various morphometric parameters. ${ }^{7,12}$ Most have found a significant association with nuclear area and perimeter. In concurrence with other studies, we also found that these parameters could be used to differentiate between the three grades of malignant lesions, except that these 
parameters were not significant in differentiating between Grades 2 vs. 3. This might be due to the smaller number of grade $2 \&$ grade 3 tumours in our study. However, various cytological grades did not show significant differences in their values of nuclear compactness and nuclear shape factor. Cytological grading (Robinson's cytological grading) categorizes lesions based on cellular dissociation, cell size, nucleoli and their morphology. These parameters are not assessed in the nuclear morphometric studies. Therefore nuclear morphometry cannot be efficiently used to grade malignant breast lesions on cytology.

Pitfall: In our study, we observed that nuclear morphometric features of lobular carcinoma breast are ineffective in distinction from benign proliferative breast lesions. This finding was similar to the one made by Rajesh L et al in their study. The morphometric parameters of lobular carcinoma were only slightly more than those of benign lesions. This proves that image analysis can be used to distinguish lobular carcinoma from ductal carcinoma but distinction of lobular carcinoma from benign and borderline lesions is difficult. $^{8}$

\section{Conclusion}

In this era where nuclear mechano-morphometric biomarkers are being efficiently used for distinguishing benign from malignant lesions, nuclear morphometry by image analysis is gaining lot of importance. Nuclear morphometry can be used as an adjuvant to FNAC. The parameters found to be consistently useful are nuclear area, nuclear perimeter and axis ratio. Therefore nuclear morphometry aids in overcoming interobserver variability and thus improves reliability and reproducibility of cytological diagnosis.

However extensive training, calibration and standardization of the software to be used are necessary to increase the efficacy and accuracy of nuclear morphometry and also to decide the standard cut off values of morphometric parameters between benign and malignant breast lesions.

\section{References}

1. Grace T. Cytopathology. London: Mosby-Wolfe; 1997.p. 78-184.

2. Al-Kaisi N. The spectrum of the "gray zone" in breast cytology: A review of 186 cases of atypical and suspicious cytology. Acta Cytol. 1994;38:898-908.

3. Yadav H, Gill M, Srivastava D, Gupta V, Gupta S, SenR.Morphometry on Breast Cytology. Turk J Path. 2015;31(3):188-93.

4. Kashyap A, Jain M, Shukla S, Andley M. Study of nuclear morphometry on cytology specimens of benign and malignant breast lesions: A study of 122 cases. $J$ Cytol. 2017;34(1):10-15.

5. Kadadavar SS, Gundalli S, Basavaraj V, Prakashini, Manjunath GV. Role of computer assisted nuclear morphometrc analysis in grading of infiltrating ductal carcinoma of breast (NOS type) and correlation with established prognostic markers. Indian Journal of Pathology and Oncology. 2015;2(4):295-302.

6. Robinson IA, Mckee, Nicholson A, D'Arcy J, Jackson PA, et al. (1994) Prognostic value of cytological grading of fine-needle aspirates from breast carcinomas. Lancet. 343:947-949.

7. Kalhan S, Dubey S, Sharma S, Duhani S, Preeti, Dixit M. Significance of nuclear morphometry in cytological aspirates of breast masses. J Cytol. 2010;27:16-21.

8. Aggarwal G, Singh S, Marwah S, Duhan D, Mathur S, Marwah N, et al. Morphometric Analysis in Breast Lesions. A Rapid Conjunct to Intraoperative Imprint Smears. Middle East J Cancer. 2012;3:1-8.

9. Abdalla F, Boder J, Buhmeida A, Hashmi H, Elzagheid A. Nuclear morphometry in FNABs of breast disease in Libyans. Anticancer Res. 2008;28:3985-89.

10. Boon ME, Trott PA, van Kaam H, Kurver PJ, Leach A. Morphometry and cytodiagnosis of breast lesions. Virchows Arch A Pathol Anat Histol. 1982;396:9-18.

11. Parmar D, Sawke N, Sawke G K. Diagnostic application of computerized nuclear morphometric image analysis in fine needle aspirates of breast lesions. Saudi Journal of health sciences. 2015;4(1):51-55.

12. Kashyap A, Jain M, Shukla S, Andley M. Study of nuclear morphometry on cytology specimens of benign and malignant breast lesions: A study of 122 cases. $J$ Cytol. 2017;34(1): 10-15.

13. Kashyap A, Jain M, Shukla S, Andley M. Role of Nuclear Morphometry in Breast Cancer and its Correlation with Cytomorphological Grading of Breast Cancer: A Study of 64 Cases. J Cytol. 2018;35(1):41-45.

14. Laishram S, Shariff S. Role of Nuclear Morphometry in distinguishing gray areas of breast lesions. JMR. 2017;3(3):132-35.

15. Rajesh L. Automated image morphometry of lobular breast carcinoma. Anal Quant Cytol Histol. 2002;24(2):81-4.

How to cite this article: Krishnappa I, Parthiban R, Sharma A, Rani P. Significance of nuclear morphometry as a diagnostic tool in fine-needle aspirates of breast masses. Indian $\mathbf{J}$ Pathol Oncol. 2018;5(4):592-597. 